\title{
THICKNESS EVOLUTION OF THE MICROSTRUCTURAL AND OPTICAL PROPERTIES OF Si:H FILMS IN THE AMORPHOUS-TO-MICROCRYSTALLINE PHASE TRANSITION REGION
}

\author{
A. S. Ferlauto ${ }^{1}$, G. M. Ferreira ${ }^{1}$, R. J. Koval ${ }^{1}$, J. M. Pearce ${ }^{1}$, C. R. Wronski ${ }^{1}$, R. W. Collins ${ }^{1}$, \\ M. M. Al-Jassim ${ }^{2}$ and K. M. Jones ${ }^{2}$ \\ 1. The Pennsylvania State University, Materials Research Institute, Department of Physics, \\ and Center for Thin Film Devices, University Park, PA 16802 \\ 2. National Renewable Energy Laboratory, 1617 Cole Boulevard, Golden, CO 80401
}

\begin{abstract}
The ability to characterize the phase of the intrinsic (i) layers incorporated into amorphous silicon [a-Si:H] and microcrystalline silicon $[\mu \mathrm{c}-\mathrm{Si}: \mathrm{H}]$ thin film solar cells is critically important for cell optimization. In this research, a new method has been developed to extract the thickness evolution of the $\mu \mathrm{c}-\mathrm{Si} \mathrm{H}$ volume fraction in mixed phase amorphous + microcrystalline silicon $[(\mathrm{a}+\mu \mathrm{c})-\mathrm{Si}: \mathrm{H}]$ i-layers. This method is based on real time spectroscopic ellipsometry measurements performed during plasmaenhanced chemical vapor deposition of the films. In the analysis, the thickness at which crystallites first nucleate from the a-Si:H phase can be estimated, as well as the nucleation density and microcrystallite cone angle. The results show very good correlations with structural and electronic device measurements.
\end{abstract}

\section{INTRODUCTION}

In previous research, extended phase diagrams have been developed that describe the accumulated thicknesses at which different microstructural and phase transitions are observed during plasma-enhanced chemical vapor deposition (PECVD) of hydrogenated silicon (Si:H) thin films [1-3]. In such diagrams, the transition thicknesses are plotted as continuous functions of the $\mathrm{H}_{2}$-dilution gas flow ratio $\mathrm{R}=\left[\mathrm{H}_{2}\right] /\left[\mathrm{SiH}_{4}\right]$, which is the key PECVD parameter that controls the phase of the film -- from amorphous silicon (a-Si:H) at low R to microcrystalline silicon ( $\mu \mathrm{c}-\mathrm{Si}: \mathrm{H})$ at high $\mathrm{R}$.

Protocrystalline $\mathrm{Si} \mathrm{H}$ is a more ordered form of a-Si:H optimized versus $R$ for electronic devices [1]. It is deposited using the maximum possible $R$ value that can be sustained without crossing the amorphous-to-(mixedphase microcrystalline) $[\mathrm{a} \rightarrow(\mathrm{a}+\mu \mathrm{c})]$ transition boundary for the desired bulk layer thickness $d_{b}$. Because this boundary exhibits a negative slope as a function of $R$ in the $R-d_{b}$ plane, microcrystalline nuclei develop within the protocrystalline Si:H films after a critical phase-transition thickness. As a result, the growing film evolves with accumulated thickness from protocrystalline $\mathrm{Si}: \mathrm{H}$ to mixedphase $\mathrm{Si}: \mathrm{H}[(\mathrm{a}+\mu \mathrm{c})-\mathrm{Si}: \mathrm{H}]$, and eventually to single-phase $\mu \mathrm{c}-\mathrm{Si}: \mathrm{H}$. Owing to the importance of $\mathrm{Si}: \mathrm{H}$ deposition near the $a \rightarrow(a+\mu c)$ and $(a+\mu c) \rightarrow \mu c$ boundaries of the phase diagram for the optimization of the intrinsic (i) layers in a-Si:H and $\mu \mathrm{c}-\mathrm{Si} \mathrm{H}$ solar cells [1-7], techniques are needed to determine the evolution of the crystalline Si:H content as a continuous function of the accumulated thickness. In this article, the application of real time spectroscopic ellipsometry (RTSE) is described for this purpose.

\section{EXPERIMENTAL DETAILS}

The Si:H films studied in this work were prepared by rf PECVD at low plasma power $\left(0.05-0.08 \mathrm{~W} / \mathrm{cm}^{2}\right)$ for use as the i-layers of a-Si:H p-i-n and $n-i-p$ solar cells. The variable parameter of greatest interest is the $\mathrm{H}_{2}$-dilution ratio $R$ which was varied over the range $0 \leq R \leq 40$. The substrate temperature for all layers was fixed at $200^{\circ} \mathrm{C}$. The Si:H films studied by RTSE were deposited in a uhvcompatible single-chamber system, whereas the solar cells for device measurements were prepared in a loadlocked multichamber system. The phase diagrams for multichamber Si:H deposition, as deduced from ex situ SE and device studies, compare well with those for singlechamber deposition, as deduced from real time SE studies [8]. Specular $\mathrm{SnO}_{2}$-coated glass served as the substrate for $p$-i-n cells, and p-layers were prepared without $\mathrm{H}_{2-}$ dilution from $\left[\mathrm{SiH}_{4}\right] /\left[\mathrm{CH}_{4}\right] /\left[\mathrm{B}\left(\mathrm{CH}_{3}\right)_{3}\right]$.

RTSE was performed using a rotating-compensator multichannel ellipsometer with a spectral range from 1.5 to $4.5 \mathrm{eV}$ and a minimum acquisition time for full spectra in $(\psi, \Delta)$ of $\sim 50 \mathrm{~ms}$ [9]. Crystalline $\mathrm{Si}(\mathrm{c}-\mathrm{Si})$ or $\mathrm{R}=0$ a-Si:H substrates were used for i-layer phase diagram studies by RTSE. The c-Si substrates provide the highest sensitivity to the microstructural and phase transitions, whereas the $\mathrm{R}=0$ a-Si:H substrates serve to simulate i-layer deposition on $\mathrm{p}$-layers for $\mathrm{p}$-i-n devices. 


\section{RESULTS AND DISCUSSION}

\section{Review Of Phase Diagrams}

First, extended phase diagrams for Si:H PECVD will be reviewed. These diagrams are developed based on RTSE analyses that extract the surface roughness layer thickness $d_{s}$ versus the bulk layer thickness $d_{b}$ from measurements performed during film deposition. Second, the optimization of a-Si:H p-i-n solar cells will be described as directed by the appropriate phase diagram [1-3].

Figure 1 shows $d_{s}$ versus $d_{b}$ for (a) two single-phase a-Si:H i-layers, one prepared at $\mathrm{R}=0$ and the other at $\mathrm{R}=10$, and (b) an i-layer prepared at $\mathrm{R}=20$ that evolves through a-Si:H, $(\mathrm{a}+\mu \mathrm{c})-\mathrm{Si}: \mathrm{H}$, and $\mu \mathrm{c}-\mathrm{Si}: \mathrm{H}$ growth regions. For these three depositions the substrates are c-Si wafers. The weak roughening onset at $d_{b} \sim 250 \AA$ for the $R=0$ a-Si:H film in Fig. 1(a) corresponds to a surface morphological transition versus $d_{b}$, but without a change in the phase of the growing film. Thus, the transition is denoted as "a $\rightarrow \mathrm{a}$ ". In this case, a simple two-layer (bulk/roughness) optical model with a uniform bulk layer was applied to analyze the RTSE data set. For the $R=20$ film in Fig. 1(b), the roughening onset at $d_{b} \sim 300 \AA$ corresponds to the $a \rightarrow(a+\mu c)$ transition, and the smoothening onset near $d_{b} \sim 1900 \AA$ corresponds to the $(\mathrm{a}+\mu \mathrm{c}) \rightarrow \mu \mathrm{c}$ transition. For this film, an optical model with a graded bulk layer was applied to analyze the RTSE data set, as will be described in greater detail in the second part of this section. For the $\mathrm{R}=10$ deposition in Fig. 1(a), a stable surface is observed after initial nuclei coalescence (i.e., for $d_{b}>100 \AA$ ), indicating that any roughening onset due to the $a \rightarrow a$ or the $a \rightarrow(a+\mu c)$ transitions must occur for $d_{b}>4000 \AA$, i.e., greater than the maximum thickness of the i-layers used in a-Si:H solar cells. Thus, the RTSE data analysis for this deposition proceeded in the same way as that for the $\mathrm{R}=0$ deposition.

The extended phase diagram is depicted in Fig. 2 for $\mathrm{Si}$ :H growth on $\mathrm{c}-\mathrm{Si}$ wafer substrates, as deduced from results including those in Fig. 1 [3]. Figure 2 shows the bulk layer thicknesses at which the $a \rightarrow a, a \rightarrow(a+\mu c)$, and $(a+\mu c) \rightarrow \mu c$ transitions occur as functions of $R$ (lines). For $\mathrm{R}<10$, the thicknesses corresponding to the $\mathrm{a} \rightarrow \mathrm{a}$ transition provide insights into the quality of the a-Si:H, irrespective of the substrate. Larger $a \rightarrow a$ transition thicknesses correlate with higher quality a-Si:H material for the i-layers of solar cells. In fact, the highest electronic quality material is prepared in the narrow region near $R=10$ where the a-Si:H surface remains stable throughout deposition to $4000 \AA$. This a-Si:H material is described as "protocrystalline" Si:H owing to the fact that if the film were to continue accumulating, then the $a \rightarrow(a+\mu c)$ transition would eventually be traversed [1]. Furthermore, if the $\mathrm{R}=10$ deposition is performed on a single-phase $\mu \mathrm{c}-\mathrm{Si}: \mathrm{H}$ substrate (rather than on oxide-covered $\mathrm{C}-\mathrm{Si}$ as in Figs. 1 and 2), then the $\mu \mathrm{c}-\mathrm{Si}: \mathrm{H}$ phase would continue to propagate [2]. Thus, for $R \geq 10$ in Fig. 2, the phase evolution is very sensitive to the nature of the substrate, and in this region it is important to consider substrates that correspond more closely to the a-Si $i_{1-x} \mathrm{C}_{\mathrm{x}}: \mathrm{H}$ p-layer used for $p$-i-n cells (or the a-Si:H n-layer used for $n-i-p$ cells).
Figure 3 shows the corresponding extended phase diagram for an $\mathrm{R}=0$ a-Si:H substrate. This substrate is relevant when considering the optimization of i-layers for devices. However, the starting roughness on the a-Si:H substrate makes it difficult to detect the weak $a \rightarrow a$ roughening transition at low $R$ as in Figs. 1(a) and 2. As a result, this transition is not shown in Fig. 3. Fortunately, insights in this region of low $\mathrm{R}$ can be obtained from the analyses of depositions on the c-Si substrate, owing to the relative substrate independence of the a-Si:H growth at lower R. In Fig. 3, the optimum one-step i-layers for solar cells are prepared at the maximum possible $R$ value while avoiding the $a \rightarrow(a+\mu c)$ transition for the desired thickness. For an i-layer thickness of $4000 \AA$, this optimum corresponds to $\mathrm{R} \sim 10$, the stable surface growth condition in Figs. 1 and 2. Figure 3 also demonstrates how optimum two-step i-layers $4000 \AA$ thick can be designed for $p-i-n$ cells on the basis of the phase diagram (see arrows) [1]. These steps include a $200 \AA \mathrm{R}=40$ i-layer at the interface to the p-layer, followed by a $3800 \AA$ thick bulk $\mathrm{i}$-layer with $\mathrm{R}=10$. This two-step $\mathrm{i}$-layer provides the highest product of open-circuit voltage $\left(\mathrm{V}_{\mathrm{oc}}\right)$ and fill factor (FF) in both the annealed and fully light-soaked states (see Fig. 4).

It is important to note, however, that the application of a single phase boundary as in Fig. 3 for guiding the twostep i-layer is an oversimplification. In fact, upon deposition of the $\mathrm{R}=40$ i-layer, the phase boundary for the subsequent $R=10$ i-layer deposition is shifted to somewhat lower $\mathrm{R}$ compared with that for the $\mathrm{R}=0$ substrate film. This effect arises owing to the substrate dependence of the phase boundary. The $\mathrm{R}=40$ layer is more ordered than the $R=0$ layer, and this promotes the $a \rightarrow(a+\mu c)$ transition at lower $d_{b}$ for fixed $R$. The $R=10$ value for the second step i-layer is sufficiently low to avoid being affected by this shift.

Figure 4 depicts $V_{O c}$ and $F F$, the latter in annealed (circles) and degraded (100 hr AM1.5; squares) states, for $\mathrm{Si} H \mathrm{H}$-i-n solar cells incorporating one-step (4000 $\AA$ ) ilayers plotted as a function of i-layer $R$ (open symbols) and for cells incorporating two-step (200 $\AA$ / $3800 \AA)$ interface/bulk i-layers plotted as a function of interface ilayer R (solid symbols) [1]. The vertical lines denote the $\mathrm{R}$ values corresponding to the $a \rightarrow(a+\mu c)$ transitions for the bulk i-layer with $d_{b}=4000 \AA$ (left) and for the interface ilayer with $d_{b}=200 \AA$ (right). The decreases in $V_{\text {oc }}$ and FF that occur for the one- and two-step i-layers for $R$ above these transition lines are attributed to the development of microcrystallinity in the surface and bulk i-layers with increasing thickness.

\section{Analysis of the evolution of $(a+\mu c)-S i: H$ films}

Once the film traverses the phase boundary from the protocrystalline $\mathrm{Si} \mathrm{H}$ growth regime into the mixed-phase $(\mathrm{a}+\mu \mathrm{c})-\mathrm{Si}: \mathrm{H}$ growth regime, the two-layer [(uniform bulk)/(surface roughness)] optical model used for analysis of the RTSE data for the $\mathrm{R}=0$ and 10 depositions of Fig. 1 (a) is no longer correct. In order to extract the volume fraction of $\mu \mathrm{c}-\mathrm{Si}: \mathrm{H}$ in the mixed-phase growth region, it is easiest to track the dielectric function of the top-most 


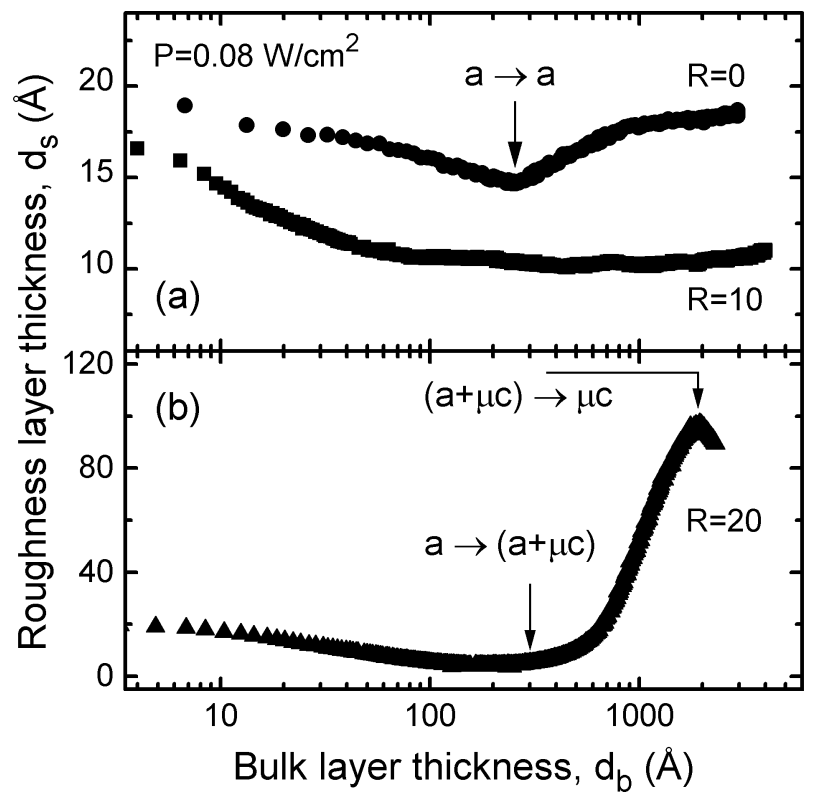

Fig. 1 Surface roughness layer thickness $\left(d_{s}\right)$ versus bulk layer thickness $\left(d_{b}\right)$ from RTSE data collected during the deposition of (a) a-Si:H with $\mathrm{R}=0$ and 10 , and (b) evolving $\mathrm{Si}: H$ with $\mathrm{R}=20$, all on $\mathrm{c}-\mathrm{Si}$ substrates.

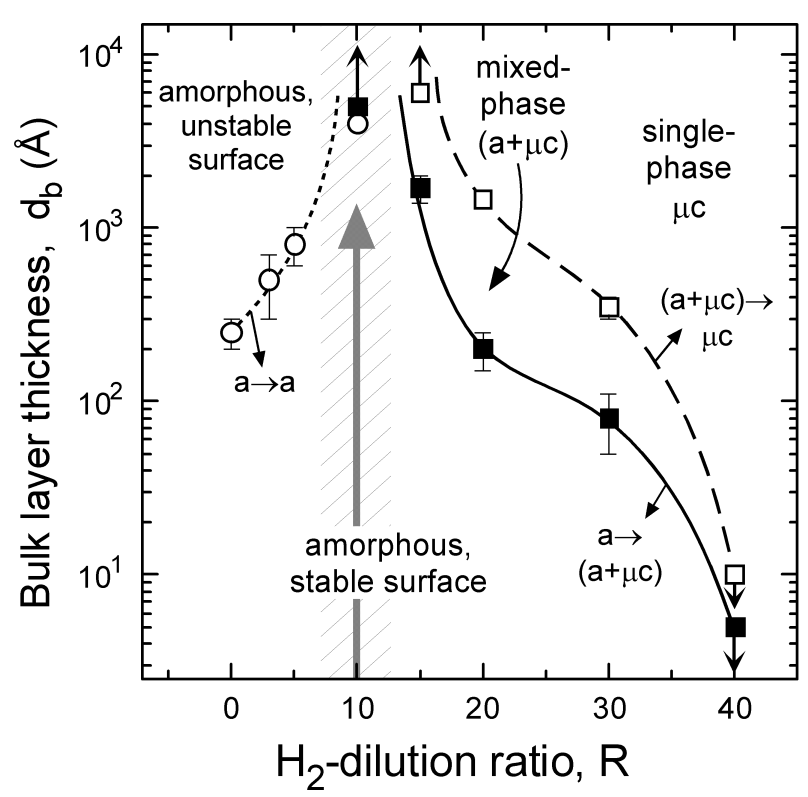

Fig. 2 Extended phase diagram in the plane of $R$ and $d_{b}$ for Si:H PECVD at a low if plasma power of $P=0.08$ $\mathrm{W} / \mathrm{cm}^{2}$. The substrates are $\mathrm{C}-\mathrm{Si}$ wafers held at $200^{\circ} \mathrm{C}$. The dotted, solid, and dashed lines identify the $a \rightarrow a$, $a \rightarrow(a+\mu c)$, and $(a+\mu c) \rightarrow \mu c$ transitions, respectively. The area between the solid and dashed lines is the mixedphase growth region of interest in this study.

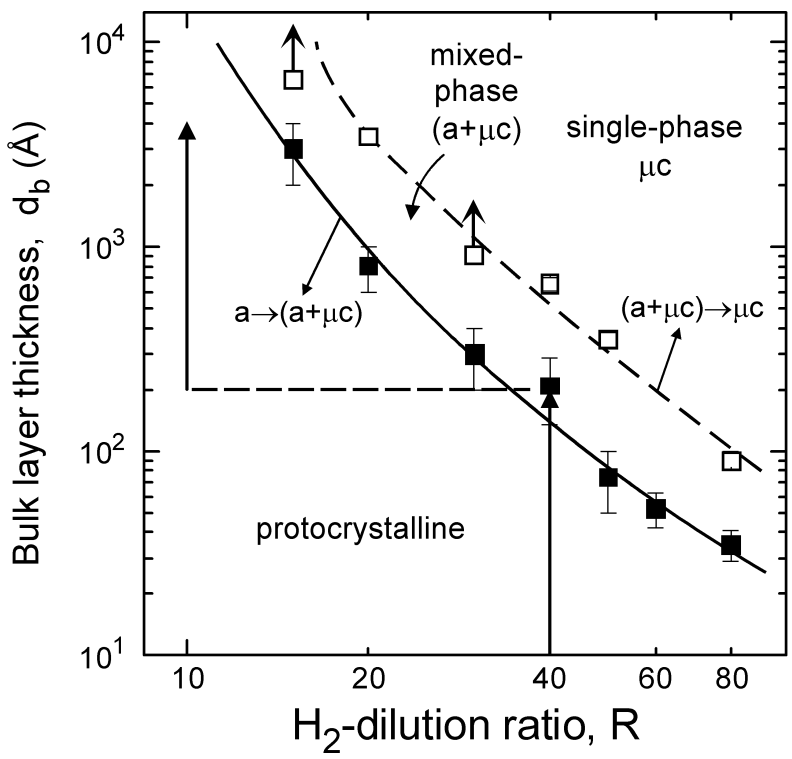

Fig. 3 Extended phase diagram in the plane of $R$ and $d_{b}$ for Si:H depositions with $\mathrm{R} \geq 10$ at a low rf plasma power of $P=0.08 \mathrm{~W} / \mathrm{cm}^{2}$. The substrates are $\mathrm{R}=0 \mathrm{a}-\mathrm{Si}: \mathrm{H}$ layers held at $200^{\circ} \mathrm{C}$

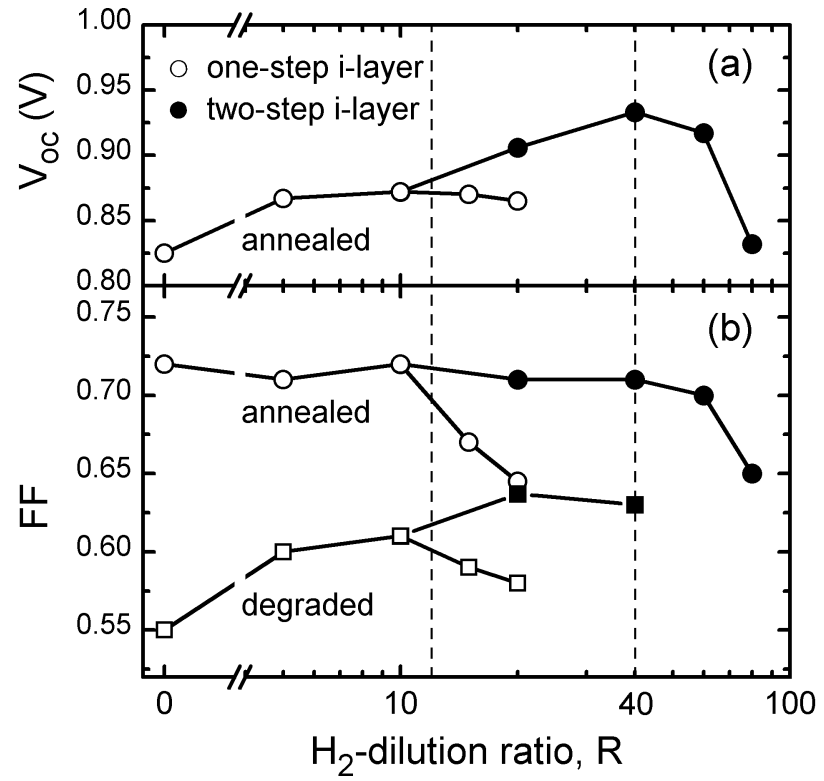

Fig. 4 (a) Open circuit voltage $V_{\text {oc }}$ and (b) fill factor FF in annealed (circles) and degraded (100 hr AM1.5; squares) states for $\mathrm{Si}: \mathrm{H}$ p-i-n solar cells incorporating onestep $(4000 \AA$ ) i-layers versus the i-layer $R$ value (open symbols), and for cells incorporating two-step (200 $\AA$ / $3800 \AA$ ) interface/bulk i-layers versus the interface i-layer $R$ value (solid symbols). 
growing material throughout the film deposition. This is an ideal application for the virtual interface (VI) approach to RTSE data analysis [10]. Figures 5-7 show the final results of such an analysis.

Figure 5 compares the (a) real and (b) imaginary parts of the dielectric functions $\left(\varepsilon_{1}, \varepsilon_{2}\right)$ for the pure phases obtained in analyses of RTSE data for the $R=20$ deposition of Fig. 1(b) on the c-Si substrate. The dielectric function of the pure a-Si:H phase was determined from the RTSE data collected in the protocrystalline Si:H growth region $0<d_{b}<200 \AA$, before the onset of the $a \rightarrow(a+\mu c)$ transition. The dielectric function of the pure $\mu \mathrm{c}-\mathrm{Si}: \mathrm{H}$ phase was obtained from data collected after crystallite contact during coalescence $\left(d_{b}>1900 \AA\right)$. In this latter case, the top $\sim 200 \AA$ of the film was characterized assuming a two-layer (bulk/roughness) model using a pseudo-substrate for the underlying graded layer. The correct surface roughness layer thickness in this model is determined by applying a global error minimization routine within which the entire VI analysis is embedded. The differences between the two dielectric functions in Fig. 5 are clear and provide support for the interpretation of the surface roughness evolution in Fig. 1(b) in terms of phase transitions. The solid lines in Fig. 5 represent best fit analytical models for the dielectric functions [11].

Figure 6 shows the evolution of the volume fraction of $\mu \mathrm{c}-\mathrm{Si}: \mathrm{H}$ in the $\mathrm{R}=20$ film of Fig. 5 throughout the growth region starting from the $a \rightarrow(a+\mu c)$ transition and ending near the $(\mathrm{a}+\mu \mathrm{c}) \rightarrow \mu \mathrm{c}$ transition. In the $\mathrm{VI}$ analysis used to deduce these results, a two-layer model similar to that of the previous paragraph is assumed. This model consists of a pseudo-substrate that incorporates the complicated history of deposition, a $\sim 30 \AA$ thick outerlayer modeled as a mixture of a-Si:H and $\mu \mathrm{c}-\mathrm{Si}: \mathrm{H}$ of variable volume fractions $1-f_{\mu c}$ and $f_{\mu c}$, respectively, and a surface roughness layer modeled as a mixture of the outerlayer material and void with fixed volume fractions of $1-f_{\mathrm{sv}}$ and $\mathrm{f}_{\mathrm{sv}}$, respectively. The dielectric function of the pseudosubstrate is determined as its pseudo-dielectric function (corrected for surface roughness, but not for the underlying graded structure). The dielectric functions of the two layers are determined from their component volume fractions and dielectric functions via the Bruggeman effective medium theory. Apparently, $d_{s}$ in Fig. 1(b) provides higher sensitivity to the presence of microcrystallites, since it leads to an $a \rightarrow(a+\mu c)$ transition of $\sim 300 \AA$, whereas the $\mu \mathrm{c}-\mathrm{Si}: \mathrm{H}$ volume fraction in Fig. 6 first extends above zero for $d_{b} \sim 700 \AA$. The solid line in Fig. 6 is the result for $f_{\mu c}$ versus $d_{b}$ employing the cone model to be described next.

Figure 7(a) reproduces the data of Fig. 1(b), and Fig. 7 (b) identifies how the microcrystallite cone model is constructed. In this model, it is assumed that all microcrystalline nuclei originate at the $a \rightarrow(a+\mu c)$ transition layer thickness ( $\sim 300 \AA$, in this case). The area density of such nuclei is assumed to be $N_{d}$, and the nuclei are assumed to grow preferentially at the expense of the a-Si:H phase with a constant, thickness-independent cone angle, $\theta$. The cones are assumed to be spherically capped whereby the cap radius $r$ evolves with bulk layer thickness according to $r=d_{b}-d_{b}$,trans. Applying this geometry, $\mathrm{N}_{\mathrm{d}}$ and $\theta$ can be deduced from the values of $\Delta \mathrm{d}_{\mathrm{b}}=\mathrm{d}_{\mathrm{b} \text {,coal }}-\mathrm{d}_{\mathrm{b} \text {,trans }}$ and $\Delta \mathrm{d}_{\mathrm{s}}=\mathrm{d}_{\mathrm{s} \text {,coal }}-\mathrm{d}_{\mathrm{s} \text {, trans. Here, }}$, $d_{b \text {,trans }}$ and $d_{s, \text { trans }}$ are the bulk and surface roughness layer thicknesses at the $a \rightarrow(a+\mu c)$ transition and $d_{b, c o a l}$ and $d_{s, c o a l}$ are the corresponding values at the $(a+\mu c) \rightarrow \mu c$ transition. For the deposition of Fig. 7(a), values of $\theta=19^{\circ}$ and $\mathrm{N}_{\mathrm{d}}=1.1 \times 10^{10} \mathrm{~cm}^{-2}$ are determined.

Figure $8(\mathrm{a})$ shows a cross-sectional transmission electron microscopy (XTEM) image from the same sample of Figs. 5-7, but at a different probe area. The cone geometry is clearly evidenced with the only major difference from the model being the statistical distribution of cones. One particular cone on the XTEM image is highlighted to depict the cone angle, and the positions of nuclei are highlighted by dots. From such measurements of the image, $\theta=25^{\circ}$ and $N_{d}=1.8 \times 10^{10} \mathrm{~cm}^{-1}$ are determined, the latter by assuming an electron beam penetration depth of $\sim 750 \AA$. These results are in good agreement with those from RTSE, as shown by the tabulation in Fig. 8(b).

Although Figs. 5-8 were obtained for a Si:H film deposited on $\mathrm{c}-\mathrm{Si}$ to make connection with the phase diagram of Fig. 2, similar analyses are possible for films deposited on $\mathrm{R}=0$ a-Si:H. In this latter case, the connection is to be made with the phase diagram of Fig. 3. Then comparisons with $\mathrm{Si}: \mathrm{H}$ electronic device performance are possible as long as the doped layers in the devices are amorphous films prepared near $\mathrm{R}=0$. As an example, Fig. 9 shows results for the dark and light current-voltage (J-V) characteristics for two Si:H p-i-n solar cells with two-step i-layers (points) [8]. Cell A employed the optimum two-step i-layer process of Fig. 4 with $R=40$ for the first $200 \AA$ of the $i$-layer and $R=10$ for the remaining $3800 \AA$. In contrast, for cell $D$, the $R=40$ layer was extended to $400 \AA$ whereas the remaining $3600 \AA$ of the ilayer was deposited at $R=20$. This latter two-step i-layer exhibited the $a \rightarrow(a+\mu c)$ transition after $200 \AA$ of $R=40$ deposition in accordance with Fig. 3 , and the $(a+\mu \mathrm{c}) \rightarrow \mu \mathrm{c}$ transition occurred after $950 \AA$ i-layer deposition.

Computer simulations of the $\mathrm{J}-\mathrm{V}$ characteristics for cells $A$ and $D$ were also carried out, and the results are shown in Fig. 9 as the solid lines. Cell $A$ could be considered in terms of a uniform a-Si:H i-layer with a mobility gap of $1.86 \mathrm{eV}$. Cell $\mathrm{D}$ on the other hand was modeled assuming a $400 \AA$ thick a-Si:H i-layer at the p/i interface with the same mobility gap as cell $A$ and a 3600 A thick $\mu \mathrm{c}-\mathrm{Si}: \mathrm{H}$ bulk i-layer with a mobility gap of $1.15 \mathrm{eV}$. In the device modeling, the a-Si:H and $\mu \mathrm{c}-\mathrm{Si}: \mathrm{H}$ phases were assumed to have similar gap state densities, and the band offsets at the $a / \mu c$ heterojunction were assumed to be split evenly between the band edges [12]. This simple model for the solar cells reproduces the overall features of the data, and in particular the voltages of the inflection points in both J-V curves. The discrepancy obtained in the simulation of $V_{\text {oc }}$ for cell $D$ is likely to be due to the oversimplification of the anisotropy of the sample arising from the one-dimensional simulation.

Table 1 includes a comparison of the RTSE and electronic simulation results for three $\mathrm{Si}: \mathrm{H} \mathrm{p}$-i-n solar cells with two-step $(R=40) /(R=20) 4000 \AA$ i-layers. The cells are distinguished by the thickness $d_{i}$ of the $R=40$ interface 


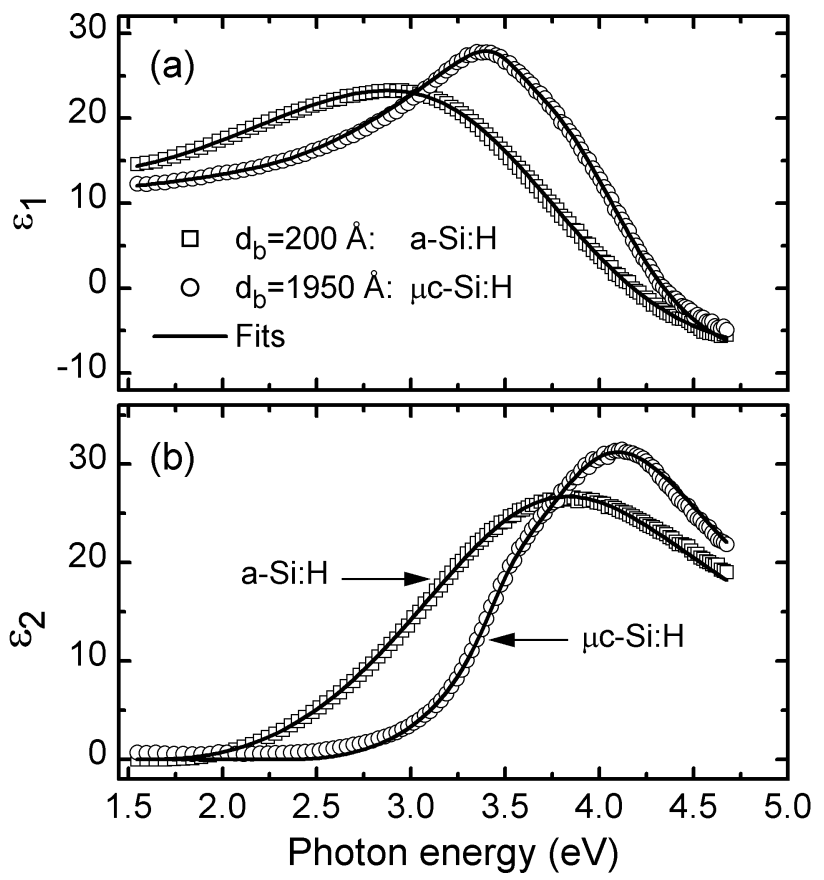

Fig. 5 (a) Real and (b) imaginary parts of the dielectric functions of the pure phases for the $\mathrm{R}=20 \mathrm{Si}: \mathrm{H}$ deposition on c-Si from Fig. 1(b). The solid lines are fits using analytical models for the dielectric functions.

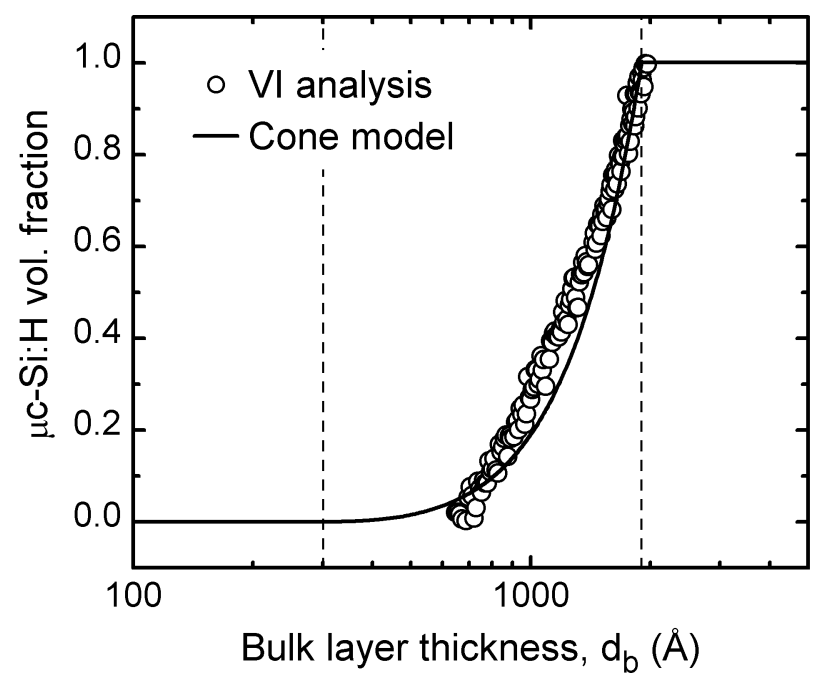

Fig. 6 Depth profile in the volume fraction of the microcrystalline phase throughout the mixed phase $(a+\mu c)-S i: H$ growth region for the $\mathrm{R}=20 \mathrm{Si} H$ deposition on c-Si from Fig. 5 (points). Also shown as the solid line is the prediction of the microcrystallite cone growth model of Fig. 7.

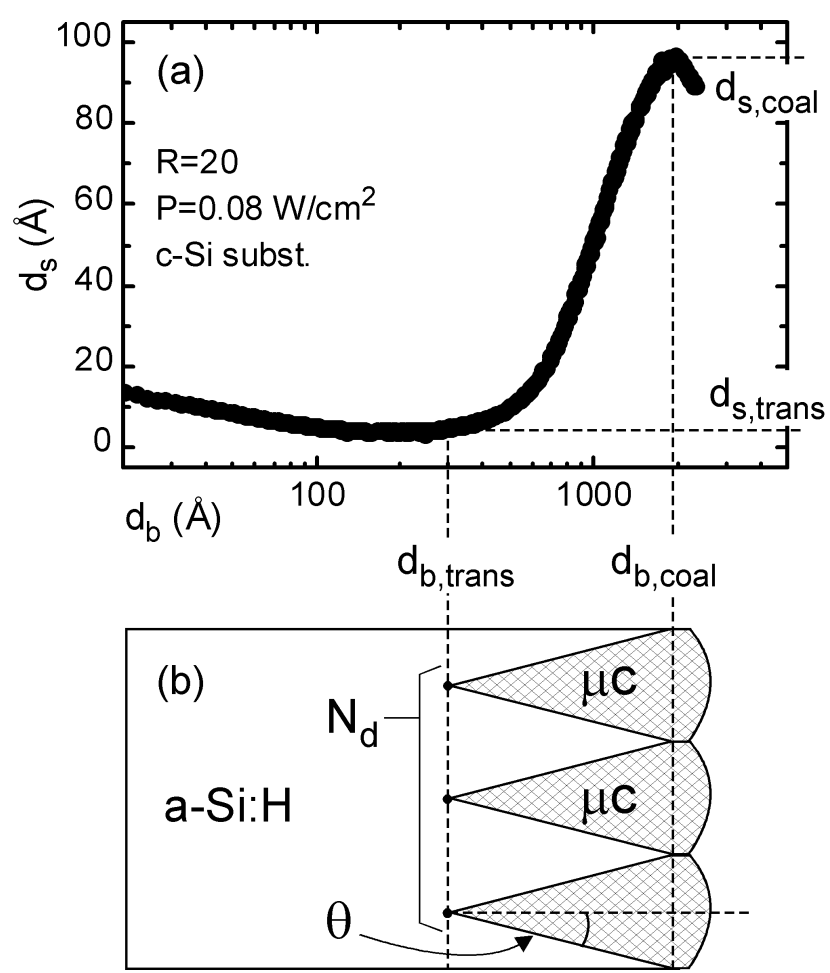

Fig. 7 (a) Surface roughness thickness versus bulk layer thickness for the $\mathrm{R}=20 \mathrm{Si}: \mathrm{H}$ deposition of Figs. 5 and 6 ; (b) schematic of the cone growth model used to estimate the microcrystallite nuclei density and cone angle.

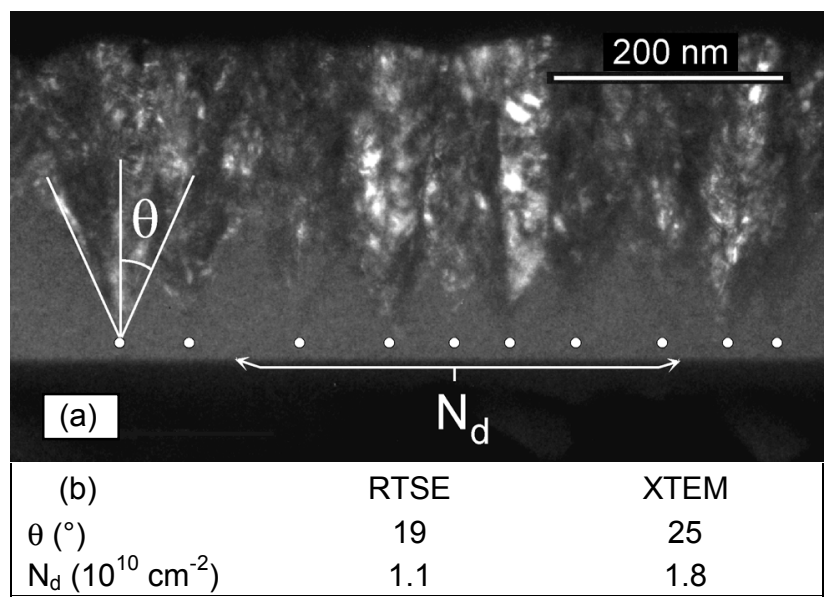

Fig. 8 (a) Cross-sectional transmission electron microscopy image for the final $\mathrm{R}=20 \mathrm{Si}: \mathrm{H}$ deposition of Figs. 5-7; (b) comparison of estimates of the microcrystallite nucleation densities and cone angles from XTEM and from RTSE. 

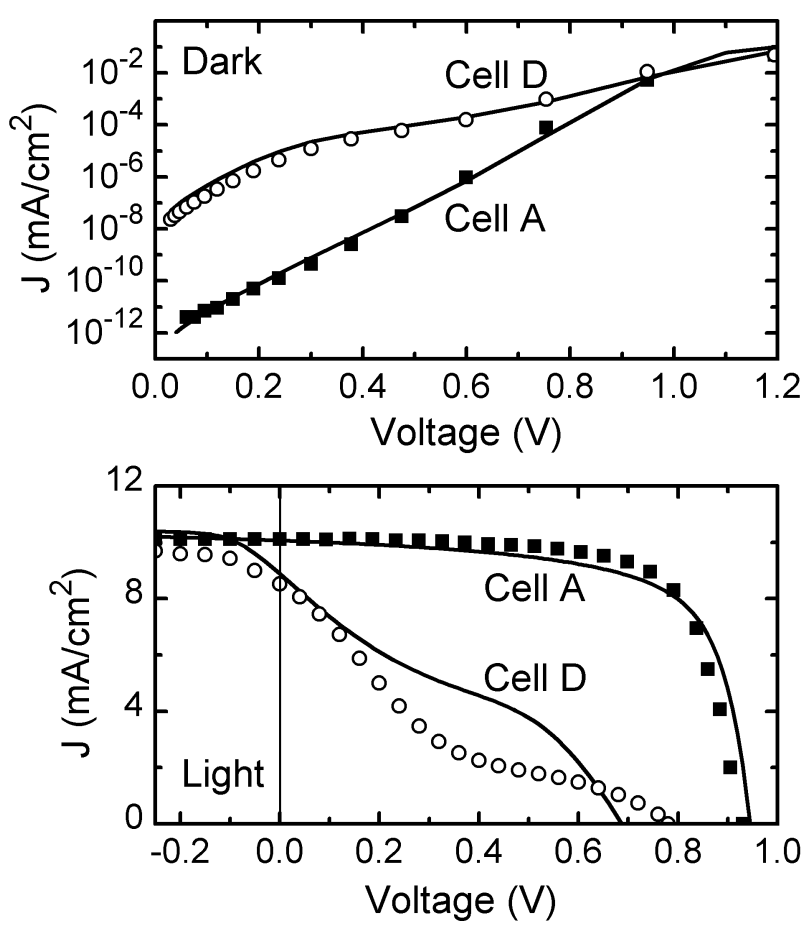

Fig. 9 Experimental (symbols) and simulated (lines) J-V characteristics for two Si:H p-i-n solar cells. Cell A incorporates the optimized $[R=40(200 \AA)] /[R=10(3800 \AA)]$ a-Si:H i-layer, and cell $D$ incorporates a $[R=40$ (400 $\AA)] /[R=20(3600 \AA)] \mathrm{Si}: \mathrm{H}$ i-layer.

i-layer. The table compares the thickness of the a-Si:H i-layer component nearest the $\mathrm{p} / \mathrm{i}$ interface, as deduced from the device modeling, with the i-layer thickness required to reach a microcrystalline volume fraction of $f_{\mu c}=0.2$ as deduced from the RTSE. This value was chosen because it gave the best overall agreement with the device modeling results (which exhibit quite large error bars due to correlation in the simulations). Good agreement can be noted between the device simulations and RTSE results suggesting that once $f_{\mu c}$ approaches $\sim 0.2$, transport is dominated by the microcrystalline phase.

\section{SUMMARY}

Real time spectroscopic ellipsometry (RTSE) measurements of plasma-enhanced chemical vapor deposition of Si:H films that undergo an amorphous-to(mixed-phase amorphous + microcrystalline) transition were performed. Novel approaches for RTSE data analysis were conceived and developed that provide (i) the nucleation density and cone angle characterizing the evolution of the Si microcrystallites in the mixed-phase amorphous + microcrystalline $(\mathrm{a}+\mu \mathrm{c})-\mathrm{Si}: \mathrm{H}$ region; and (ii) the volume fraction of the $\mu \mathrm{c}-\mathrm{Si}: \mathrm{H}$ phase as a function of the accumulated thickness, as well as (iii) the optical properties of the films in the amorphous, mixed-phase $(\mathrm{a}+\mu \mathrm{c})-\mathrm{Si}: \mathrm{H}$, and single-phase $\mu \mathrm{c}-\mathrm{Si}: \mathrm{H}$ growth regions. The resulting information correlates well with ex situ structural and device performance measurements.
Table 1. Correlation of RTSE and device modeling results for $p$-i-n cells with $4000 \AA$ two-step $R=40 / 20$ i-layers in which the thickness of the $R=40$ interface layer is varied.

\begin{tabular}{|c|c|c|}
\hline $\begin{array}{l}\text { First step: } \\
R=40 \\
\text { thickness }(\AA)\end{array}$ & $\begin{array}{l}\text { RTSE: } \\
d_{b} \text { when } \\
f_{\mu c}=0.2(\AA)\end{array}$ & $\begin{array}{l}\text { Device Modeling: } \\
\text { Heterojunction } \\
\text { position from p/i }(\AA)\end{array}$ \\
\hline 0 & $1240 \pm 30$ & $1300 \pm 300$ \\
\hline 150 & $950 \pm 30$ & $700 \pm 200$ \\
\hline 400 (cell D) & $330 \pm 20$ & $400 \pm 100$ \\
\hline
\end{tabular}

\section{Acknowledgments}

The authors acknowledge support of this research by NREL under Subcontracts XAF-8-17619-22 and AAD-918-668-09 and by NSF under Grants DMR-9820170 and DMR-0137240.

\section{REFERENCES}

[1] J. Koh, Y. Lee, H. Fujiwara, C.R. Wronski, and R.W. Collins, Appl. Phys. Lett. 73, 1526 (1998).

[2] J. Koh, A.S. Ferlauto, P.I. Rovira, C.R. Wronski, and R.W. Collins, Appl. Phys. Lett. 75, 2286 (1999).

[3] A.S. Ferlauto, R.J. Koval, C.R. Wronski, and R.W. Collins, Appl. Phys. Lett. 80, 2666 (2002).

[4] D.V. Tsu, B.S. Chao, S.R. Ovshinsky, S. Guha, and J. Yang, Appl. Phys. Lett. 71, 1317 (1997).

[5] J. Yang, K. Lord, S. Guha, and S.R. Ovshinsky, Mater. Res. Soc. Symp. Proc. 609, A15.4 (2001)

[6] C. Koch, M. Ito, V. Svrcek, M.B. Schubert, and J.H. Werner, Mater. Res. Soc. Symp. Proc. 609, A15.6 (2001).

[7] O. Vetterl, F. Finger, R. Carius, P. Hapke, L. Houben, O. Kluth, A. Lambertz, A. Muck, B. Rech, and H. Wagner, Sol. Energy Mater. Sol. Cells 62, 97 (2000).

[8] R.J. Koval, J.M. Pearce, A.S. Ferlauto, R.W. Collins, and C.R. Wronski, Mater. Res. Soc. Symp. Proc. 664, A16.4 (2001).

[9] R.W. Collins, J. Koh, H. Fujiwara, P.I. Rovira, A.S. Ferlauto, J.A. Zapien, C.R. Wronski, and R. Messier, Appl. Surf. Sci. 154, 217 (2000).

[10] H. Fujiwara, J. Koh, C.R. Wronski, and R.W. Collins, Appl. Phys. Lett. 70, 2150 (1997).

[11] A.S. Ferlauto, J. Koh, P.I. Rovira, C.R. Wronski, R.W. Collins, and G. Ganguly, J. Non-Cryst. Solids 266-269, 269 (2000).

[12] E. Bohmer and H. Luth, J. Non-Cryst. Solids 266-269, 1038 (2000). 